HEPHY-PUB 957/16

UWThPh-2016-2

February 2016

\title{
ANALYTIC BETHE-SALPETER DESCRIPTION OF THE LIGHTEST PSEUDOSCALAR MESONS
}

\author{
Wolfgang LUCHA* \\ Institute for High Energy Physics, \\ Austrian Academy of Sciences, \\ Nikolsdorfergasse 18, A-1050 Vienna, Austria \\ Franz F. SCHÖBERL ${ }^{\dagger}$ \\ Faculty of Physics, University of Vienna, \\ Boltzmanngasse 5, A-1090 Vienna, Austria
}

\begin{abstract}
Within the Bethe-Salpeter formalism for instantaneous interactions, we describe, along a totally analytic route, the lightest pseudoscalar mesons by quark-antiquark bound states which show at least three indispensable general features, namely, the (almost) masslessness required for pions and kaons to be interpretable as (pseudo) Goldstone bosons, the suitable asymptotic behaviour in the limit of large spacelike relative momenta as determined by the relationship between quark mass function and Bethe-Salpeter amplitudes, and a pointwise behaviour for finite spacelike relative momenta suited for guaranteeing colour confinement.
\end{abstract}

PACS numbers: 11.10.St, 03.65.Ge, 03.65.Pm

* E-mail address: wolfgang.lucha@oeaw.ac.at

$\dagger$ E-mail address: franz.schoeberl@univie.ac.at 


\section{Introduction}

Within particle physics, the members of the multiplet of lightest pseudoscalar mesons, viz., pions and kaons, can be interpreted from two perspectives: on the one hand, they appear to be bound states of the fundamental degrees of freedom, i.e., quarks and gluons, of quantum chromodynamics (QCD), the quantum field theory which describes the strong interactions; on the other hand, they may be regarded as the nearly massless (pseudo) Goldstone bosons of the spontaneously (and, to a minor degree, explicitly) broken chiral symmetries of QCD.

Quantum field theory describes bound states by means of the covariant Bethe-Salpeter formalism [1]. The latter's instantaneous limit [2], with the Salpeter equation [3] as its most prominent outcome, enables us to evade obstacles arising in applications of this framework. Recently, by use of earlier developed inversion techniques [4] we related, for Goldstone-type quark-antiquark bound states, Salpeter solutions compatible with constraints arising from QCD Dyson-Schwinger equations to configuration-space potentials $V(r), r=|\boldsymbol{x}|$, encoding the impacts of strong interactions $[5,6]$. In this paper, we implement, in addition, boundary conditions imposed by colour confinement on the solutions of the Bethe-Salpeter equation.

The outline of this paper is as follows. In Sec. 2, we present a rather condensed sketch of our actual route from the Bethe-Salpeter equation to the interaction potentials responsible for the formation of light pseudoscalar mesons acting as pseudo-Goldstone bosons of QCD. In Sec. 3, we briefly recall how to exploit solutions of the Dyson-Schwinger equation for the quark propagator to derive information about the behaviour of meson Salpeter amplitudes. In Sec. 4, we considerably refine the outcomes of Ref. [6] by taking into account a particular implication of the violation of the axiom of reflection positivity - which acts as a sufficient but not necessary condition for confinement - for the propagator of any coloured degree of freedom of QCD. In Sec. 5, we discuss some of those cases that allow for analytic derivation of the potentials. Finally, in Sec. 6 we collect the insights gained by this sharpened analysis.

\section{Instantaneous Bethe-Salpeter Handling of Mesons}

\subsection{The Salpeter approach to fermion-antifermion bound states}

Within the framework of relativistic quantum field theories, the bound states of elementary particles - with total momentum $P$ and relative momentum $p$ - can be described by their Bethe-Salpeter amplitude $\Phi(p, P)$, governed by the homogeneous Bethe-Salpeter equation [1]. If the interactions between the bound-state constituents may be approximated by their instantaneous limit and if the propagators of these particles depend on the time component $p_{0}$ of $p$ in a sufficiently simple form, this Bethe-Salpeter equation reduces, upon integration over $p_{0}$, to a (generic) instantaneous Bethe-Salpeter equation [2] for the Salpeter amplitude

$$
\phi(\boldsymbol{p}) \equiv \frac{1}{2 \pi} \int \mathrm{d} p_{0} \Phi(p) .
$$

Assuming, for the bound-state constituents, free propagation with effective masses leads to the Salpeter equation [3]. Defining, for the particle $i=1,2$ of mass $m_{i}$ and momentum $\boldsymbol{p}$, by

$$
E_{i}(\boldsymbol{p}) \equiv \sqrt{\boldsymbol{p}^{2}+m_{i}^{2}}, \quad H_{i}(\boldsymbol{p}) \equiv \gamma_{0}\left(\boldsymbol{\gamma} \cdot \boldsymbol{p}+m_{i}\right), \quad \Lambda_{i}^{ \pm}(\boldsymbol{p}) \equiv \frac{E_{i}(\boldsymbol{p}) \pm H_{i}(\boldsymbol{p})}{2 E_{i}(\boldsymbol{p})},
$$

its free energy $E_{i}(\boldsymbol{p})$, Dirac Hamiltonian $H_{i}(\boldsymbol{p})$ and projection operators $\Lambda_{i}^{ \pm}(\boldsymbol{p})$ for positive or negative energy, this Salpeter equation, for the bound states of a fermion of mass $m_{1}$ and 
momentum $\boldsymbol{p}_{1}$ and an antifermion of mass $m_{2}$ and momentum $\boldsymbol{p}_{2}$, can be cast into the form

$$
\begin{aligned}
\phi(\boldsymbol{p})=\int \frac{\mathrm{d}^{3} q}{(2 \pi)^{3}} & \left(\frac{\Lambda_{1}^{+}\left(\boldsymbol{p}_{1}\right) \gamma_{0}[K(\boldsymbol{p}, \boldsymbol{q}) \phi(\boldsymbol{q})] \gamma_{0} \Lambda_{2}^{-}\left(\boldsymbol{p}_{2}\right)}{P_{0}-E_{1}\left(\boldsymbol{p}_{1}\right)-E_{2}\left(\boldsymbol{p}_{2}\right)}\right. \\
& \left.-\frac{\Lambda_{1}^{-}\left(\boldsymbol{p}_{1}\right) \gamma_{0}[K(\boldsymbol{p}, \boldsymbol{q}) \phi(\boldsymbol{q})] \gamma_{0} \Lambda_{2}^{+}\left(\boldsymbol{p}_{2}\right)}{P_{0}+E_{1}\left(\boldsymbol{p}_{1}\right)+E_{2}\left(\boldsymbol{p}_{2}\right)}\right) .
\end{aligned}
$$

The integration kernel, $K(\boldsymbol{p}, \boldsymbol{q})$, subsumes the interactions experienced by the bound-state constituents. If the fermions couple identically, its action on $\phi(\boldsymbol{q})$ forms a series of products of tensor products $\Gamma \otimes \Gamma$ of matrices $\Gamma$ in Dirac space and Lorentz-scalar potentials $V_{\Gamma}(\boldsymbol{p}, \boldsymbol{q})$ :

$$
[K(\boldsymbol{p}, \boldsymbol{q}) \phi(\boldsymbol{q})]=\sum_{\Gamma} V_{\Gamma}(\boldsymbol{p}, \boldsymbol{q}) \Gamma \phi(\boldsymbol{q}) \Gamma .
$$

The energy projectors in Eq. (2) entail for all solutions (1) the (in fact, single [7]) constraint

$$
\Lambda_{1}^{+}\left(\boldsymbol{p}_{1}\right) \phi(\boldsymbol{p}) \Lambda_{2}^{+}\left(\boldsymbol{p}_{2}\right)=\Lambda_{1}^{-}\left(\boldsymbol{p}_{1}\right) \phi(\boldsymbol{p}) \Lambda_{2}^{-}\left(\boldsymbol{p}_{2}\right)=0 .
$$

Evidently, such three-dimensional reduction enables us to construct a relationship between (Poincaré-covariant) descriptions of bound states by means of the Bethe-Salpeter equation and the notion of static interaction potentials acting between the bound-state constituents.

\section{$2.2 \quad$ Light pseudoscalar mesons}

The Salpeter amplitude $\phi(\boldsymbol{p})$ of any bound state composed of a spin- $\frac{1}{2}$ fermion and a spin- $\frac{1}{2}$ antifermion with vanishing total spin quantum number involves, upon expansion over some basis in the "Dirac" space of complex $4 \times 4$ matrices, just two independent components. Let us call them $\varphi_{1}(\boldsymbol{p})$ and $\varphi_{2}(\boldsymbol{p})$. The general form of a Salpeter amplitude $\phi(\boldsymbol{p})$ is determined by the constraint (4). It can be read off from, e.g., Eq. (4.9) of Ref. [8] or Eq. (12) of Ref. [9]; for the bound states of interest, only three terms enter into such "Dirac" expansion of $\phi(\boldsymbol{p})$. For simplicity, we focus, in the following to the flavour-symmetric limit of bound states of a quark and an antiquark of equal masses $m_{1}=m_{2}=m$. Then, recalling the definition (2) of the Dirac Hamiltonian, two of the three terms merge to $H(\boldsymbol{p})$, and all $\phi(\boldsymbol{p})$ acquire the form

$$
\phi(\boldsymbol{p})=\left[\varphi_{1}(\boldsymbol{p}) \frac{H(\boldsymbol{p})}{E(\boldsymbol{p})}+\varphi_{2}(\boldsymbol{p})\right] \gamma_{5}
$$

It is not an extremely daring move to assume the interaction kernel to be of convolution type and to respect spherical symmetry. In this case, clearly, the trivial reference to angular variables may be separated from the dependence on the radial momentum variables $p \equiv|\boldsymbol{p}|$ etc., the Salpeter equation (3) can be reduced to an equivalent system of coupled equations for the radial factors $\varphi_{i}(p)$ of the independent Salpeter components $\varphi_{i}(\boldsymbol{p}), i=1,2, \ldots$, and the effective interactions may be described by central potentials $V_{\Gamma}(r)$, each of which enters the radial equations by its Fourier-Bessel transforms [8,9]. Dropping the index $\Gamma$, the latter read, in terms of the spherical Bessel functions of the first kind $[10] j_{i}(z), i=0, \pm 1, \pm 2, \ldots$,

$V_{L}(p, q) \equiv 8 \pi \int_{0}^{\infty} \mathrm{d} r r^{2} j_{L}(p r) j_{L}(q r) V(r), \quad p \equiv|\boldsymbol{p}|, \quad q \equiv|\boldsymbol{q}|, \quad L=0,1,2, \ldots$

We intend to infer (or, at least, to constrain) the potential functions $V_{\Gamma}(\boldsymbol{p}, \boldsymbol{q})$ from some knowledge of the solutions $\phi(\boldsymbol{p})$ of the Salpeter equation (3). In order to pose a well-defined inversion problem, the Lorentz nature of the Dirac-matrix tensor products $\Gamma \otimes \Gamma$ entering in one's interaction kernel $K(\boldsymbol{p}, \boldsymbol{q})$ must be specified. As done in Refs. [5,6], we adopt for $\Gamma \otimes \Gamma$ 
the Fierz-symmetric linear combination of scalar, pseudoscalar and vector Dirac structures

$$
\Gamma \otimes \Gamma=\frac{1}{2}\left(\gamma_{\mu} \otimes \gamma^{\mu}+\gamma_{5} \otimes \gamma_{5}-1 \otimes 1\right)
$$

Apart from its phenomenological importance, the advantage of this choice is the collapse of the Salpeter equation (3) for a spin-singlet bound state to two coupled eigenvalue equations for the two radial components, $\varphi_{1}(p)$ and $\varphi_{2}(p)$, determining the Salpeter amplitude (5) [7]:

$$
\begin{aligned}
& 2 E(p) \varphi_{2}(p)+2 \int_{0}^{\infty} \frac{\mathrm{d} q q^{2}}{(2 \pi)^{2}} V_{0}(p, q) \varphi_{2}(q)=\widehat{M} \varphi_{1}(p), \\
& 2 E(p) \varphi_{1}(p)=\widehat{M} \varphi_{2}(p), \quad E(p) \equiv \sqrt{p^{2}+m^{2}} .
\end{aligned}
$$

For all bound states with mass eigenvalues $\widehat{M} \equiv \sqrt{P^{2}}=0$ - and thus, in particular, for every Goldstone boson, owing to its inevitably vanishing mass - these equations decouple: the second relation (being of purely algebraic nature) forces $\varphi_{1}(p)$ to vanish identically, i.e., $\varphi_{1}(p) \equiv 0$, whereas the other one - an integral equation equivalent to the spinless Salpeter equation $^{1}$ - governs, via $\varphi_{2}(p)$, the Salpeter amplitude of any massless spin-singlet meson:

$$
E(p) \varphi_{2}(p)+\int_{0}^{\infty} \frac{\mathrm{d} q q^{2}}{(2 \pi)^{2}} V_{0}(p, q) \varphi_{2}(q)=0 .
$$

Thus, the general form of each spin-singlet solution $\phi(\boldsymbol{p})$ corresponding to a vanishing mass eigenvalue $\widehat{M}$ of the Salpeter equation (3) with Lorentz structure of its interaction kernel of the Fierz-symmetric form $\Gamma \otimes \Gamma=\frac{1}{2}\left(\gamma_{\mu} \otimes \gamma^{\mu}+\gamma_{5} \otimes \gamma_{5}-1 \otimes 1\right)$ is given by $\phi(\boldsymbol{p})=\varphi_{2}(\boldsymbol{p}) \gamma_{5}$. So, the actual task is to solve the Salpeter equation (7) for known potential function $V_{0}(p, q)$ or, by inversion, to extract the underlying potential $V(r)$ from knowledge of its solution $\varphi_{2}(q)$.

\subsection{Configuration-space inversion of bound-state problem}

Introducing, in terms of the spherical Bessel function of the first kind $j_{0}(z)=(\sin z) / z[10]$, the Fourier-Bessel transforms of Salpeter component $\varphi_{2}(p)$ and kinetic term $E(p) \varphi_{2}(p)$ by

$$
\varphi(r) \equiv \sqrt{\frac{2}{\pi}} \int_{0}^{\infty} \mathrm{d} p p^{2} j_{0}(p r) \varphi_{2}(p), \quad T(r) \equiv \sqrt{\frac{2}{\pi}} \int_{0}^{\infty} \mathrm{d} p p^{2} j_{0}(p r) E(p) \varphi_{2}(p),
$$

out of the system of coupled relations forming the Bethe-Salpeter quintessence (6) the only member that "survives" the Goldstone limit $\widehat{M} \rightarrow 0$, Eq. (7), reads, in configuration space,

$$
T(r)+V(r) \varphi(r)=0 .
$$

From such bound-state equation, the potential $V(r)$ may be read off by division by $\varphi(r)[6]$ :

$$
V(r)=-\frac{T(r)}{\varphi(r)} .
$$

Of course, some caution must be exercised if the Salpeter amplitude in configuration space, $\varphi(r)$, exhibits one or more zeros, since, in general, each such zero will induce a singularity of the potential $V(r)$ : for instance, if $\varphi(r)$ proves to have a single zero at $r=r_{0}>0$ one might be well advised to first consider the domain $\left(0, r_{0}\right) \cup\left(r_{0}, \infty\right)$ and then take the limits $r \rightarrow r_{0}$. It goes without saying that such due care is implicitly understood in the following analyses.

\footnotetext{
${ }^{1}$ For concise reviews elucidating various facets of the spinless Salpeter equation, consult, e.g., Refs. [11].
} 


\section{Quark Propagator Constrains Salpeter Amplitudes}

In the present context, both foundation and primary source of information for constraining the behaviour of the Salpeter amplitude (1) as a function of the relative momentum $\boldsymbol{p}$ is the observation $[12,13]$ that, in the chiral limit, the renormalized axial-vector Ward-Takahashi identity of QCD relates the solution of the Bethe-Salpeter equation for a flavour-nonsinglet pseudoscalar meson to the solution of the Dyson-Schwinger equation for the dressed quark propagator $S(p)$, defined by two (real) Lorentz-scalar functions which can be interpreted as the quark mass function, $M\left(p^{2}\right)$, and quark wave-function renormalization function, $Z\left(p^{2}\right)$ :

$$
S(p)=\frac{\mathrm{i} Z\left(p^{2}\right)}{\not p-M\left(p^{2}\right)+\mathrm{i} \varepsilon}, \quad \not p \equiv p^{\mu} \gamma_{\mu}, \quad \varepsilon \downarrow 0 .
$$

From this relationship, we may conclude [6] that, in Euclidean-space formulation, indicated by underlined coordinates, the Bethe-Salpeter amplitudes of massless pseudoscalar mesons in the center-of-momentum frame $(\underline{\boldsymbol{P}}=0)$ are controlled by $M\left(\underline{k}^{2}\right)$ and $Z\left(\underline{k}^{2}\right)$ according to

$$
\Phi(\underline{k}, 0) \propto \frac{Z\left(\underline{k}^{2}\right) M\left(\underline{k}^{2}\right)}{\underline{k}^{2}+M^{2}\left(\underline{k}^{2}\right)} \underline{\gamma}_{5}+\text { subleading contributions . }
$$

Ignoring the comparatively minor variation of $Z\left(\underline{k}^{2}\right)$ with $\underline{k}^{2}$ leads to the sought relation [6]

$$
\Phi(\underline{k}, 0) \propto \frac{M\left(\underline{k}^{2}\right)}{\underline{k}^{2}+M^{2}\left(\underline{k}^{2}\right)} \underline{\gamma}_{5}+\cdots .
$$

In Ref. [6], we used as our first piece of information about $M\left(\underline{k}^{2}\right)$ a kind of by-product of a QCD-compatible model calculation [13] of the quark propagator $S(\underline{k})$ in Euclidean space, viz. that in the chiral limit the quark mass function decays, for large $\underline{k}^{2}$, essentially like $\underline{k}^{-2}$ :

$$
\lim _{\underline{k}^{2} \rightarrow \infty} M\left(\underline{k}^{2}\right) \propto \frac{1}{\underline{k}^{2}} \quad \Longrightarrow \quad \lim _{\underline{k}^{2} \rightarrow \infty} \Phi(\underline{k}, 0) \propto \lim _{\underline{k}^{2} \rightarrow \infty} \frac{M\left(\underline{k}^{2}\right)}{\underline{k}^{2}} \underline{\gamma}_{5} \propto \frac{1}{\underline{k}^{4}} \underline{\gamma}_{5} .
$$

Introducing a parameter $\mu$ with the dimension of mass, and guided by our preference for an analytic treatment if feasible, we modelled the above feature of $\Phi(\underline{k}, 0)$ by the simple ansatz

$$
\Phi(\underline{k}, 0)=\frac{1}{\left(\underline{k}^{2}+\mu^{2}\right)^{2}} \underline{\gamma}_{5} .
$$

Integration w.r.t. the Euclidean-time $\underline{k}$ component gave, for the Salpeter component $\varphi_{2}(p)$,

$$
\varphi_{2}(p)=4 \sqrt{\frac{\mu^{3}}{\pi}} \frac{1}{\left(p^{2}+\mu^{2}\right)^{3 / 2}}, \quad \mu>0, \quad\left\|\varphi_{2}\right\|^{2} \equiv \int_{0}^{\infty} \mathrm{d} p p^{2}\left|\varphi_{2}(p)\right|^{2}=1,
$$

which, in configuration space, is just the modified Bessel function of second kind $K_{0}(z)[10]$ :

$$
\varphi(r)=\frac{4 \sqrt{2 \mu^{3}}}{\pi} K_{0}(\mu r), \quad \mu>0, \quad\|\varphi\|^{2} \equiv \int_{0}^{\infty} \mathrm{d} r r^{2}|\varphi(r)|^{2}=1 .
$$

For the kinetic term $T(r)$ and the potential $V(r)$, this ansatz yields [6], e.g., for $\mu=m>0$,

$$
T(r)=\frac{2 \sqrt{2 m^{3}}}{r} \exp (-m r), \quad V(r)=-\frac{\pi}{2} \frac{\exp (-m r)}{r K_{0}(m r)} .
$$

In the following, as our evident next step we improve the somewhat naïve ansatz (11) by accommodating, in addition to the quark mass function's asymptotic behaviour considered above, the needs of colour confinement as disclosed by axiomatic quantum field theory [14]. 


\section{Consequences of the Axiom of Reflection Positivity}

Having taken advantage from our knowledge of the ultraviolet behaviour of the quark mass function, logically our next move must be to fathom the implications of colour confinement for any potential $V(r)$ specifying an interaction kernel $K(\boldsymbol{p}, \boldsymbol{q})$ of the Salpeter equation (3).

\subsection{Confinement and analytic properties of Schwinger functions}

From the point of view of experiment, colour confinement forms a just heuristic description of the empirically established fact of non-observation of isolated coloured particles, i.e., the absence of the fundamental coloured degrees of freedom of quantum chromodynamics from the spectrum of observable states. Within the framework of quantum field theory, a precise definition of colour confinement may be formulated $[15,16]$ in terms of Schwinger functions, specific (not time-ordered) analytic $n$-point functions of field operators in Euclidean space, by analytic continuation of the Wightman functions of axiomatic quantum field theory [14]. By the Osterwalder-Schrader reconstruction theorem [17], each Schwinger function related to an element of the Hilbert space of observables fulfills the axiom of reflection positivity. In other words, compliance with the axiom of reflection positivity forms a necessary condition for such relationship of Schwinger functions to elements of the Hilbert space of observables.

For a two-point Schwinger function, the fulfilment of the axiom of reflection positivity is equivalent to the existence of a Källén-Lehmann representation of this Schwinger function. This, in turn, forbids inflexion points of this Schwinger function at spacelike momenta [16]. Phrased the other way round, a two-point Schwinger function with any such inflexion point violates the axiom of reflection positivity and therefore cannot correspond to an element in the Hilbert space of observables: the respective degree of freedom is subject to confinement.

Our aim is to confine quarks, with mass function $M\left(\underline{k}^{2}\right)$, inside a Goldstone-type meson with bound-state mass $\widehat{M}=0$, that is, to prevent the quarks from entering the spectrum of observables by hindering them to propagate to infinity. In other words, we must assure that a quark propagator is not the propagator of an observable particle. According to the above, one circumstance which guarantees this is the violation of the axiom of reflection positivity by the (Euclidean-space) quark two-point Schwinger function, connected to the propagator associated to the quark in Minkowski space. Now, the Källén-Lehmann representation of a Schwinger function severely constrains its momentum-space behaviour in a variety of ways. Among others, it does not tolerate the presence of an inflexion point at spacelike momenta. Hence, a possibility to achieve such confinement-enforcing breach of the axiom of reflection positivity is to assume for the two-point Schwinger function a behaviour incompatible with at least one of the simple constraints imposed on the analytical properties of any two-point Schwinger function by the sheer fact of its possession of a Källén-Lehmann representation. Of course, confinement may originate from other roots or reveal itself in a different manner.

For the purpose of the present investigation, we would like to place our full wager on the occurrence of an inflexion point. Following an admittedly rather heuristic line of argument, let us assume that the confinement-promoting properties of the quark two-point Schwinger function will be carried over to the quark propagator (9) in Euclidean-space representation and let us, tentatively, attribute the latter characteristics to some appropriate behaviour of the quark mass function $M\left(\underline{k}^{2}\right)$. Then, by Eq. (10), resulting from the relationship between (two-point) quark propagator and (three-point) quark-meson vertex implied $[12,13]$ by the axial-vector Ward-Takahashi identity of QCD, such facets will eventually get imprinted on the Bethe-Salpeter amplitude $\Phi(\underline{k}, 0)$ of the Goldstone-type quark-antiquark bound state. 


\subsection{Implementation of some consequences of colour confinement}

In view of the fundamental interrelationships, compendiously sketched in Sec. 4.1, between the qualitative pointwise behaviour of propagators in momentum-space representation and the occurrence of confinement of the associated elementary excitations, we now consider an ansatz for the Bethe-Salpeter amplitude compatible with the requirements of confinement.

Clearly, by way of our starting point, Eq. (10), ensuring colour confinement by asserting the existence of an inflexion point in the quark mass function $M\left(\underline{k}^{2}\right)$ will be reflected by the pointwise behaviour of the associated Bethe-Salpeter amplitude $\Phi(\underline{k}, \underline{P})$. Therefore, in the center-of-momentum frame of the bound state, all these considerations, combined with our bias towards analytic manageability, suggest as ansatz for $\Phi(\underline{k}, 0)$ of still rather simple form

$$
\Phi(\underline{k}, 0)=\left[\frac{1}{\left(\underline{k}^{2}+\mu^{2}\right)^{2}}+\frac{\eta \underline{k}^{2}}{\left(\underline{k}^{2}+\mu^{2}\right)^{3}}\right] \underline{\gamma}_{5}, \quad \mu>0, \quad \eta \in \mathbb{R} .
$$

An integration with respect to the momentum component $\underline{k}_{4}$ yields the Salpeter amplitude

$$
\phi(\boldsymbol{k}) \equiv \frac{1}{2 \pi} \int \mathrm{d} \underline{k}_{4} \Phi(\underline{k})=\frac{4(1+\eta) \boldsymbol{k}^{2}+(4+\eta) \mu^{2}}{16\left(\boldsymbol{k}^{2}+\mu^{2}\right)^{5 / 2}} \underline{\gamma}_{5} \propto \varphi_{2}(|\boldsymbol{k}|) \underline{\gamma}_{5}
$$

and thus, according to Eq. (5), the normalized Salpeter function $\varphi_{2}(p)$ we are interested in:

$$
\varphi_{2}(p)=\sqrt{\frac{256 \mu^{3}}{\pi[256+\eta(320+109 \eta)]}} \frac{4(1+\eta) p^{2}+(4+\eta) \mu^{2}}{\left(p^{2}+\mu^{2}\right)^{5 / 2}}, \quad\left\|\varphi_{2}\right\|^{2}=1 .
$$

So, in momentum space our Salpeter component exhibits a smooth behaviour. It assumes a (for all $\eta$ real) finite value at the origin $p=0$, and vanishes, of course, in the limit of large $p$ :

$$
\varphi_{2}(0)=\frac{16(4+\eta)}{\sqrt{\pi[256+\eta(320+109 \eta)] \mu^{3}}}, \quad \varphi_{2}(p) \underset{p \rightarrow \infty}{\longrightarrow} 0 .
$$

In configuration-space representation, this Salpeter-component ansatz is expressible by means of two modified Bessel functions $K_{n}(z)$ of the second kind [10] of the orders $n=0,1$ :

$$
\varphi(r)=\frac{16}{\pi} \sqrt{\frac{2 \mu^{3}}{256+\eta(320+109 \eta)}}\left[4(1+\eta) K_{0}(\mu r)-\eta \mu r K_{1}(\mu r)\right], \quad\|\varphi\|^{2}=1 .
$$

The two specific values $\eta=0$ and $\eta=-1$ of the mixing parameter constitute critical points of the inversion formalism utilized here [4] in the sense that for these two exceptional values no longer an interplay of the two contributions to the right-hand side of Eq. (14) but rather the first or second term alone determines the behaviour of the configuration-space Salpeter component function $\varphi(r)$, and therefore nature and shape of the interaction potential $V(r)$. Accordingly, in all following claims these two critical values of $\eta$ deserve separate attention.

Obviously, $\varphi(r)$ diverges at the origin $r=0$, except for $\eta=-1$, and vanishes for large $r$ :

$$
\begin{array}{ll}
\varphi(0)=\frac{16}{3 \pi} \sqrt{\frac{2 \mu^{3}}{5}} & \text { for } \eta=-1, \\
\varphi(r) \underset{r \rightarrow 0}{\longrightarrow}-\frac{64(1+\eta)}{\pi} \sqrt{\frac{2 \mu^{3}}{256+\eta(320+109 \eta)} \ln (\mu r) \underset{r \rightarrow 0}{\longrightarrow} \infty} & \text { for } \eta \neq-1 ; \\
\varphi(r) \underset{r \rightarrow \infty}{\longrightarrow} 0 . &
\end{array}
$$


Due to the exponential decay of the modified Bessel functions of the second kind $K_{n}(z)$, $n=0,1,2, \ldots$, the configuration-space Salpeter component $\varphi(r)$ given by Eq. (14) has one, and only one, zero, $r_{0}$, for each choice of the mixing parameter $\eta$ lying in one of the intervals $-\infty<\eta \supsetneqq-1$ or $0 \supsetneqq \eta<\infty$. Table 1 lists the numerical value of this zero (in units of $1 / \mu$ ) for several choices of $\eta$ considered in the following. Barring the rather unlikely possibility of an accidental zero of the kinetic term $T(r)$ at the same location $r=r_{0}$, the division by $\varphi(r)$ required by Eq. (8) forces the zero $r_{0}$ to induce a singularity of the potential $V(r)$ at $r=r_{0}$.

Table 1: Zero $r_{0}$ of the Salpeter component (14) for $\mu=1$ and various mixing parameters $\eta$.

\begin{tabular}{lr}
\hline \hline$\eta$ & $r_{0}[1 / \mu]$ \\
\hline 0.5 & $11.51002562 \ldots$ \\
1 & $7.51478467 \ldots$ \\
1.5 & $6.18423766 \ldots$ \\
2 & $5.51940196 \ldots$ \\
\hline \hline
\end{tabular}

\begin{tabular}{lc}
\hline \hline \multicolumn{1}{c}{$\eta$} & $r_{0}[1 / \mu]$ \\
\hline-1.25 & $0.41415074 \ldots$ \\
-1.5 & $0.90800844 \ldots$ \\
-1.75 & $1.27449577 \ldots$ \\
-2 & $1.55265125 \ldots$ \\
\hline \hline
\end{tabular}

Figure 1 shows, in appropriate units of $\mu$ (or, equivalently, for $\mu=1$ ), for a few values of the mixing parameter $\eta$, the behaviour of our inflexion-friendly ansatz for the independent Salpeter component, in both momentum space, Eq. (13), and configuration space, Eq. (14).

The only remaining challenge is to deduce the kinetic term $T(r)$. In the following, this is done for two (trivially) analytically accessible cases: $m=0$ (Sec. 5.1) and $m=\mu$ (Sec. 5.2).

\section{Analytic Configuration-Space Confining Potentials}

Fed with the ansatz (13), our inversion machinery promptly returns all potentials aimed at.

In view of our discussion of the presence of one zero $r_{0}$ in the ansatz (14) for the Salpeter component $\varphi(r)$ in Sec. 4.2, we have to expect that, for any value of the mixing parameter $\eta$ outside the "safe" interval $-1 \leq \eta \leq 0$, any potential $V(r)$ inferred in this way will develop a singularity at the location of this zero $r_{0}$. As a matter of fact, this singularity will prove to be an infinite discontinuity of $V(r)$ and $V(r)$ to exhibit one of the following two behaviours:

$$
\begin{array}{llll}
V(r) \underset{r \rightarrow r_{0}^{-}}{\longrightarrow}+\infty, & V^{\prime}(r) \underset{r \rightarrow r_{0}^{-}}{\longrightarrow}+\infty, & V(r) \underset{r \rightarrow r_{0}^{+}}{\longrightarrow}-\infty, & V^{\prime}(r) \underset{r \rightarrow r_{0}^{+}}{\longrightarrow}+\infty ; \\
V(r) \underset{r \rightarrow r_{0}^{-}}{\longrightarrow}-\infty, & V^{\prime}(r) \underset{r \rightarrow r_{0}^{-}}{\longrightarrow}-\infty, & V(r) \underset{r \rightarrow r_{0}^{+}}{\longrightarrow}+\infty, & V^{\prime}(r) \underset{r \rightarrow r_{0}^{+}}{\longrightarrow}-\infty .
\end{array}
$$

The mixing $\widetilde{\eta}$ where $V(r)$ flips from one to the other is fixed by its competing contributions.

As a consequence, for $\eta \notin[-1,0]$, when tracing this potential $V(r)$, for $r$ rising from the origin $r=0$, one encounters an infinite discontinuity situated at $r=r_{0}$, i.e., a region where this potential $V(r)$ grows beyond bounds. Accordingly, for interquark separations $r$ smaller than the $\varphi(r)$ zero $r_{0}$, that is, in the region $0 \leq r<r_{0}$, the potential $V(r)$ can be claimed to exhibit a confining behaviour. In this spirit, the location $r_{0}$ of the singularity of $V(r)$ off the spatial origin $r=0$ might be interpreted as introducing some sort of "confinement radius." 


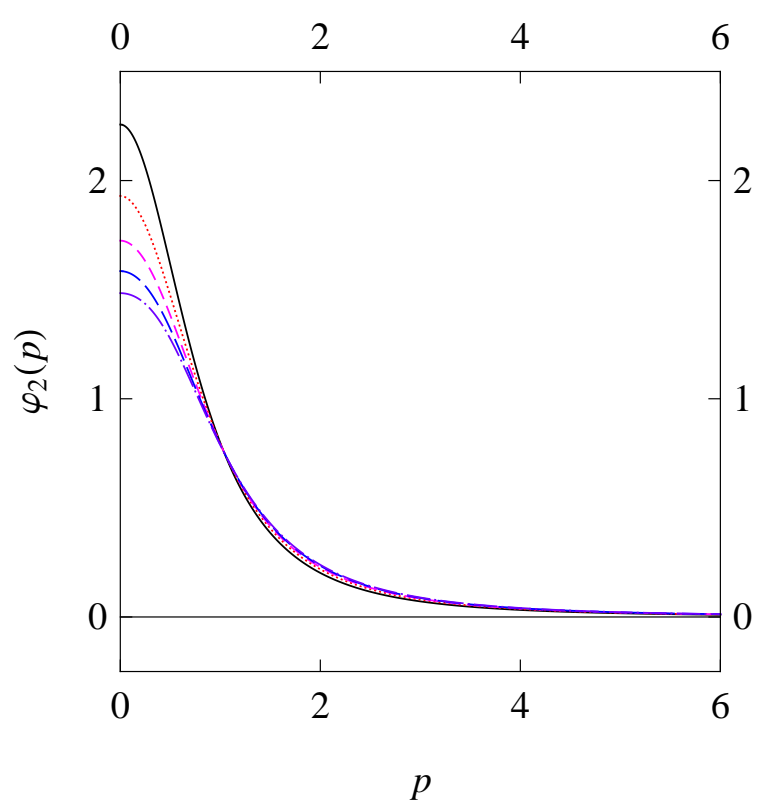

(a)

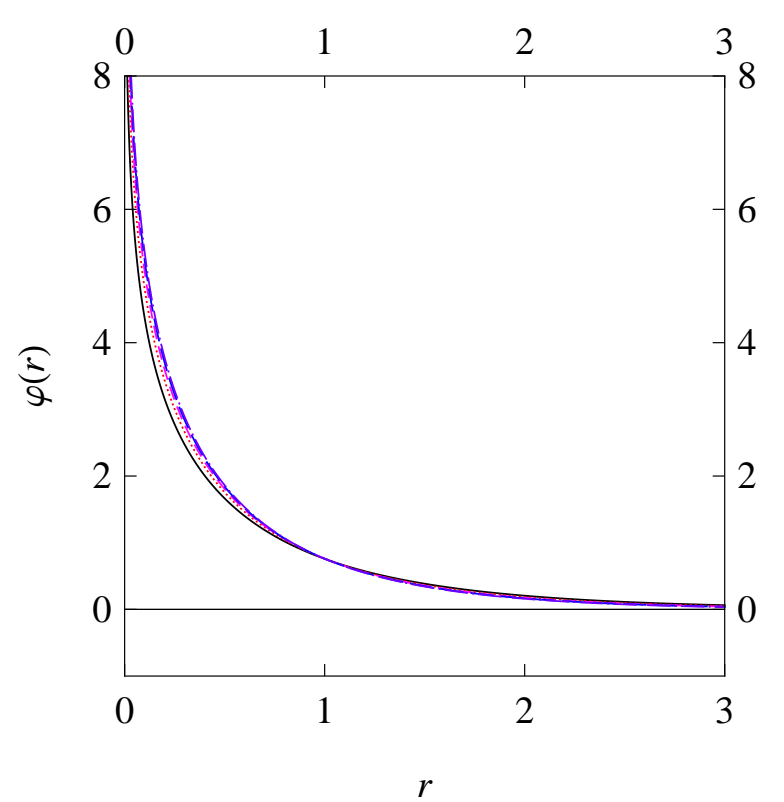

(b)

Figure 1: Independent Salpeter component fully defining, at least for any Salpeter equation (3) characterized by the Lorentz structure $2 \Gamma \otimes \Gamma=\gamma_{\mu} \otimes \gamma^{\mu}+\gamma_{5} \otimes \gamma_{5}-1 \otimes 1$ of the interaction kernel, the Salpeter amplitude (5) for pseudoscalar mesons, shown in adequate units of $\mu$ in its (a) momentum-space representation, $\varphi_{2}(p) \propto\left(p^{2}+1\right)^{-3 / 2}+\eta\left(p^{2}+\frac{1}{4}\right)\left(p^{2}+1\right)^{-5 / 2}$, and (b) configuration-space representation, $\varphi(r) \propto K_{0}(r)+\eta\left[K_{0}(r)-r K_{1}(r) / 4\right]$, at the value $\eta=0$ (black solid line [6, Sec. 4.C]), $\eta=0.5$ (red dotted line), $\eta=1$ (magenta short-dashed line), $\eta=1.5$ (blue long-dashed line) and $\eta=2$ (violet dot-dashed line) of our mixing parameter.

\subsection{Analytic treatment: bound-state constituents of mass $\boldsymbol{m}=0$}

For massless quarks, i.e., if $m=0$, the configuration-space kinetic term $T(r)$ corresponding to the Salpeter component (14) can be expressed in terms of both modified Bessel functions of the first kind [10] $I_{n}(z)$ for $n=0,1$ and modified Struve functions [10] $\mathbf{L}_{n}(z)$ for $n=0,1$ :

$$
\begin{aligned}
T(r) & =\sqrt{\frac{2 \mu^{3}}{256+\eta(320+109 \eta)}} \frac{8}{\pi r}\left\{\pi\left[4+\eta\left(4+\mu^{2} r^{2}\right)\right]\left[I_{0}(\mu r)-\mathbf{L}_{0}(\mu r)\right]\right. \\
& \left.+\pi(4+5 \eta) \mu r\left[I_{1}(\mu r)-\mathbf{L}_{1}(\mu r)\right]-4(2+3 \eta) \mu r\right\} .
\end{aligned}
$$

In the extracted interaction potential, the nasty normalization factor necessarily drops out:

$$
\begin{aligned}
V(r)=- & \left\{\pi\left[4+\eta\left(4+\mu^{2} r^{2}\right)\right]\left[I_{0}(\mu r)-\mathbf{L}_{0}(\mu r)\right]+\pi(4+5 \eta) \mu r\left[I_{1}(\mu r)-\mathbf{L}_{1}(\mu r)\right]\right. \\
& -4(2+3 \eta) \mu r\} /\left\{2 r\left[4(1+\eta) K_{0}(\mu r)-\eta \mu r K_{1}(\mu r)\right]\right\}
\end{aligned}
$$

With the relation $\pi \mathbf{L}_{1}(z)+2=\pi \mathbf{L}_{-1}(z)$ [e.g., Eq. (12.2.4) of Ref. [10] for $\nu=0$ ], it is trivial to show that, for $\eta=0$, this potential $V(r)$ reduces to that one found in Sec. V.A of Ref. [6]. At the origin $r=0$, this potential $V(r)$ develops, except for $\eta=-1$, a Coulomb singularity governed by the first term in its denominator and logarithmically softened by the divergent 
behaviour, $K_{0}(x \rightarrow 0) \approx-\ln (x)$, of the modified Bessel function of the second kind $K_{0}(x)$ :

$$
\begin{array}{ll}
V(0)=-2 \mu & \text { for } \eta=-1, \\
V(r) \underset{r \rightarrow 0}{\longrightarrow} \frac{\pi}{2 r \ln (\mu r)} \underset{r \rightarrow 0}{\longrightarrow}-\infty & \text { for } \eta \neq-1 .
\end{array}
$$

With the exception of the case $\eta=-1$, for which this potential $V(r)$ assumes a finite value, the independence of this short-distance behaviour of $V(r)$ from the parameter $\eta$ controlling the amount of admixture of the second term in our ansatz (12) renders clear the irrelevance of the second term in the denominator of $V(r)$ for the behaviour of $V(r)$ at the origin $r=0$.

For the distance $r$ rising from zero to $\infty$, the qualitative behaviour of the potential (15), exemplified in Figs. 2 through 4 in units of appropriate powers of $\mu$ (which is tantamount to setting $\mu=1$ ), varies among regions of $\eta$ separated by the critical points $\eta=0$ and $\eta=-1$ :

- For $\eta>0$ (Fig. 2), $V(r)$ rises for increasing $r$ monotonically to $\infty$ at the discontinuity at $r=r_{0}$, where it jumps from $+\infty$ to $-\infty$ and then remains below zero up to $r=\infty$.

- For $-1<\eta \leq 0$ (Fig. 3), in spite of the absence of zeros in $\varphi(r)$ in this case, $V(r)$ rises for increasing $r$ monotonically from its notorious singularity at $r=0$ to $\infty$ for large $r$.

- For $\eta \leq-1$ (Fig. 4), $V(r)$ is affected by its "switching" value of $\eta, \widetilde{\eta} \approx-1.520216412$ :

- For $\widetilde{\eta}<\eta<-1$, with rising $r V(r)$ first stays below zero up to $r_{0}$, there it jumps from $-\infty$ to $+\infty$, then passes a local minimum and finally rises to $\infty$ for large $r$.

- For $-\infty<\eta<\widetilde{\eta}$, for $r$ approaching the $\varphi(r)$-induced discontinuity from the left $V(r)$ rises to $\infty$, then jumps from $+\infty$ to $-\infty$, and rises again, for $r \rightarrow \infty$, to $\infty$.

With due satisfaction we find that, for massless quarks, the ansatz (12) defines, irrespective of the choice of the mixing $\eta$, for one reason or the other a potential capable of confinement.

\subsection{Analytic result: bound-state constituents of mass $m=\mu>0$}

In case the bound-state constituents' mass $m$ equals precisely the mass $\mu$ that parametrizes our ansatz (12), that is, for $\mu=m>0$, the numerator and denominator of the integrand in the Fourier-Bessel transform of the kinetic term $E(p) \varphi_{2}(p)$ resemble each other to a degree that enables $T(r)$ to be an analytic expression with Yukawa and exponential contributions:

$$
T(r)=\frac{4 \sqrt{2 m^{3}}[8+\eta(8-3 m r)]}{\sqrt{256+\eta(320+109 \eta)}} \frac{\exp (-m r)}{r} .
$$

Accordingly, our prototype potential for the case of massive bound-state constituents reads

$$
V(r)=-\frac{\pi[8+\eta(8-3 m r)] \exp (-m r)}{4 r\left[4(1+\eta) K_{0}(m r)-\eta m r K_{1}(m r)\right]} .
$$

Similarly to the case $m=0$ studied in Sec. 5.1, and for the same reasons, except for $\eta=-1$ the potential $V(r)$ has its logarithmically softened Coulomb singularity at the origin $r=0$ :

$$
\begin{array}{ll}
V(0)=-3 \pi m / 4=-m \times 2.35619449 \ldots & \text { for } \eta=-1, \\
V(r) \underset{r \rightarrow 0}{\longrightarrow} \frac{\pi}{2 r \ln (m r)} \underset{r \rightarrow 0}{\longrightarrow}-\infty & \text { for } \eta \neq-1 .
\end{array}
$$




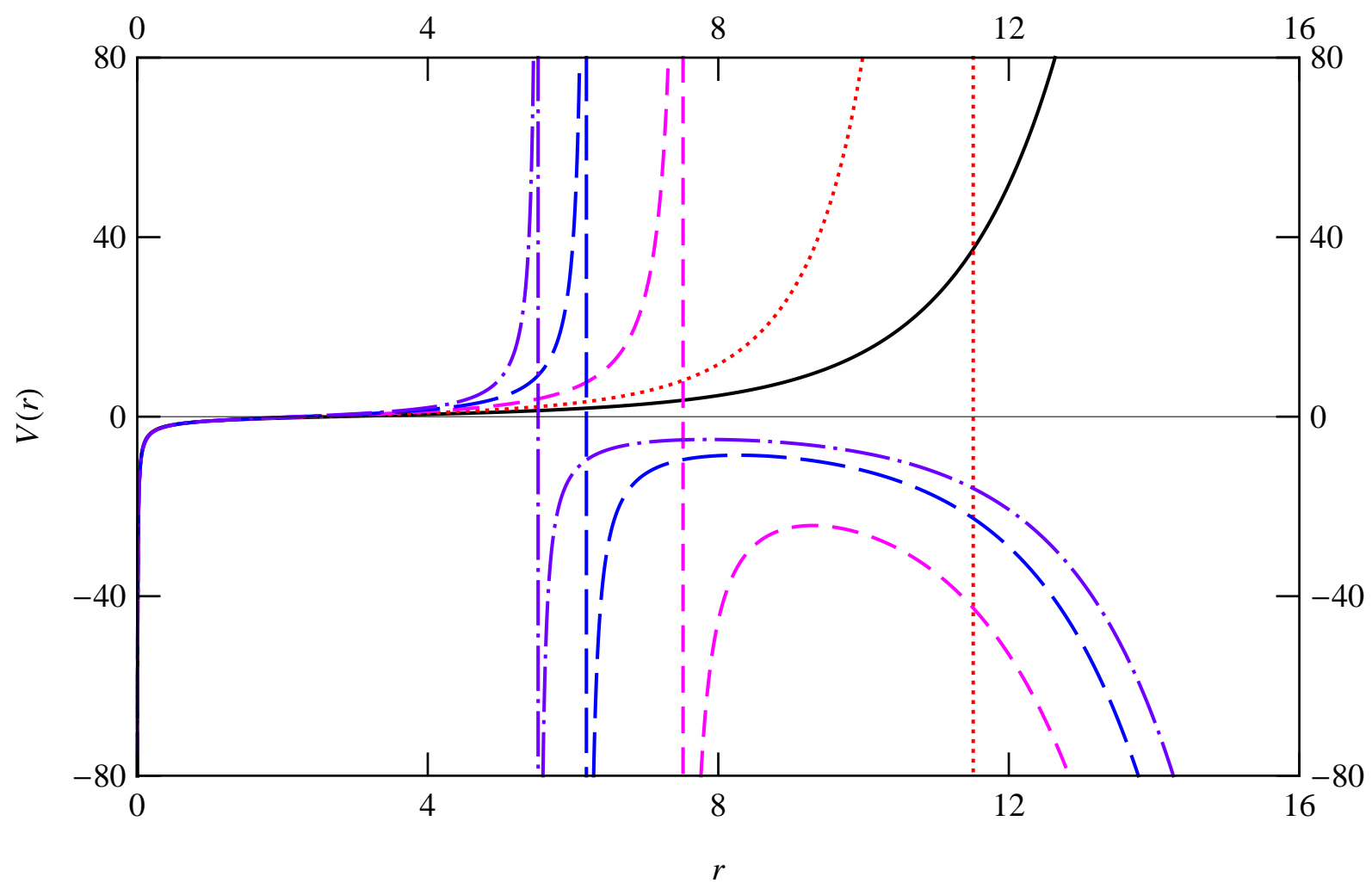

Figure 2: Configuration-space potential $V(r)$ extracted from the Salpeter equation (3) with interaction-kernel Lorentz structure $2 \Gamma \otimes \Gamma=\gamma_{\mu} \otimes \gamma^{\mu}+\gamma_{5} \otimes \gamma_{5}-1 \otimes 1$ by assuming the ansatz $\varphi_{2}(p) \propto\left(p^{2}+1\right)^{-3 / 2}+\eta\left(p^{2}+\frac{1}{4}\right)\left(p^{2}+1\right)^{-5 / 2}$ for the nonvanishing component of the Salpeter amplitude (5) to describe massless pseudoscalar bound states of fermions with mass $m=0$ : $V(r)=-N(r) / D(r)$ with the two abbreviations $D(r) \equiv 2 r\left[4(1+\eta) K_{0}(r)-\eta r K_{1}(r)\right]$ and $N(r) \equiv \pi\left[4+\eta\left(4+r^{2}\right)\right]\left[I_{0}(r)-\mathbf{L}_{0}(r)\right]+\pi(4+5 \eta) r\left[I_{1}(r)-\mathbf{L}_{1}(r)\right]-4(2+3 \eta) r$ for notational ease introduced for denominator and numerator, respectively, depicted for the values $\eta=0$ (black solid line [6, Sec. 5.A]), $\eta=0.5$ (red dotted line), $\eta=1$ (magenta short-dashed line), $\eta=1.5$ (blue long-dashed line) and $\eta=2$ (violet dot-dashed line) of our mixing parameter.

In the limit $r \rightarrow \infty$, all $V(r)$ approach zero, with decay controlled for $\eta=0$ by the modified Bessel function $K_{0}[6]$ but for $\eta \neq 0$ by the modified Bessel function $K_{1}$ in the denominator:

$$
\begin{array}{lrl}
V(r)=-\frac{\pi}{2} \frac{\exp (-m r)}{r K_{0}(m r)} \underset{r \rightarrow \infty}{\longrightarrow}-\sqrt{\frac{\pi m}{2 r}} \underset{r \rightarrow \infty}{\longrightarrow} 0 & \text { for } \eta=0[6], \\
V(r) \underset{r \rightarrow \infty}{\longrightarrow}-\frac{3 \pi}{4} \frac{\exp (-m r)}{r K_{1}(m r)} \underset{r \rightarrow \infty}{\longrightarrow}-\frac{3}{2} \sqrt{\frac{\pi m}{2 r}} \underset{r \rightarrow \infty}{\longrightarrow} 0 & \text { for } \eta \neq 0 .
\end{array}
$$

The potential (16) rises to $\infty$ in precisely those regions of $\eta$ that enforce a zero of $\varphi(r)$, as depicted, for $\mu=1$, in Figs. 5 and 6 for those cases where a confining behaviour is found:

- For $\eta>0$ (Fig. 5), $V(r)$ fulfils all confining obligations by growing beyond bounds for $r \nearrow r_{0}$, then drops from $+\infty$ to $-\infty$, and finally approaches zero in the limit $r \rightarrow \infty$.

- For $-1 \leq \eta \leq 0$, in which case $V(r)$ does not encounter a $\varphi(r)$-related singularity, the monotonic rise with $r$ to the asymptotic value $V(\infty)=0$ betrays lack of confinement. 


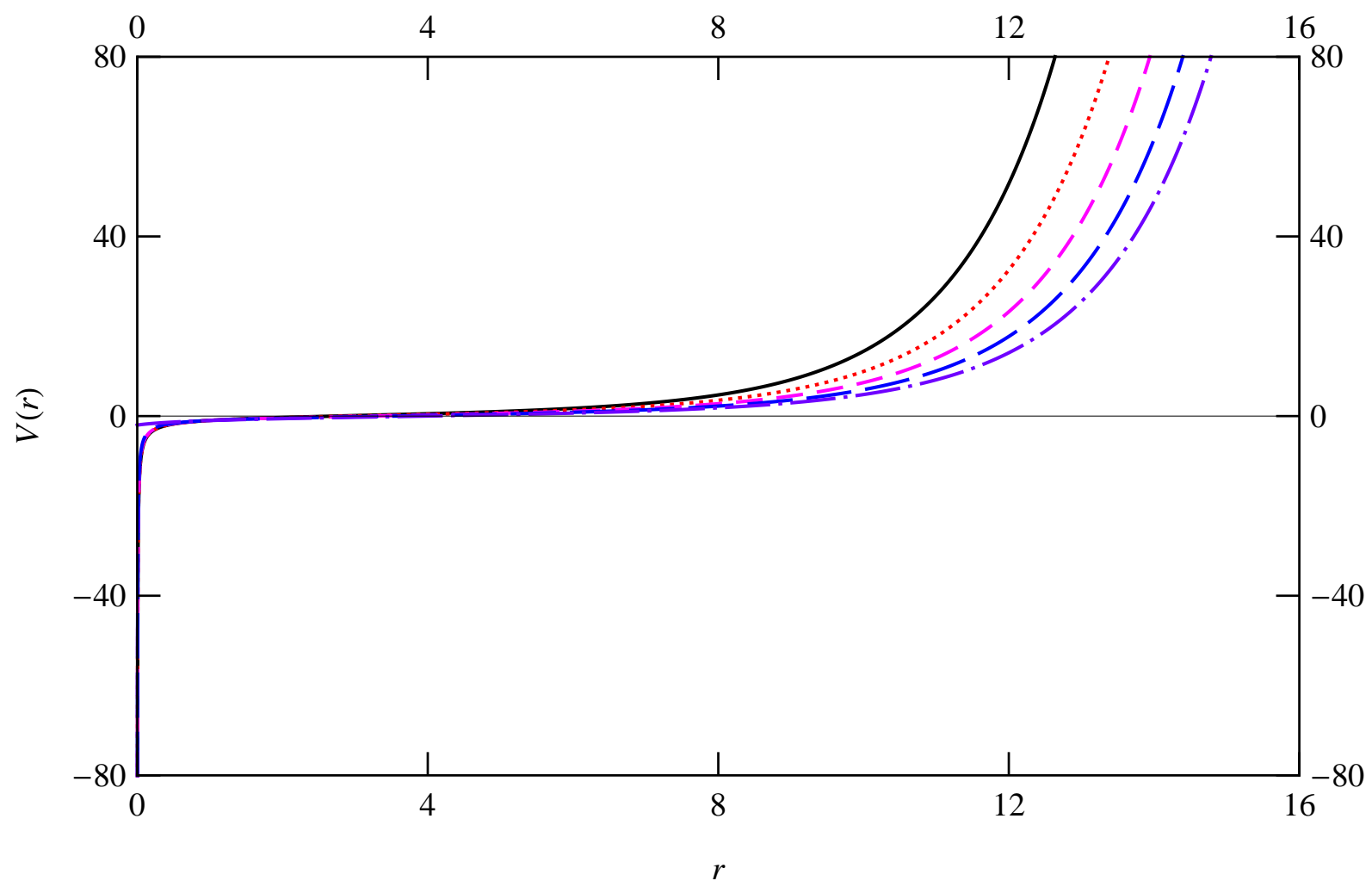

Figure 3: Configuration-space potential $V(r)$ extracted from the Salpeter equation (3) with interaction-kernel Lorentz structure $2 \Gamma \otimes \Gamma=\gamma_{\mu} \otimes \gamma^{\mu}+\gamma_{5} \otimes \gamma_{5}-1 \otimes 1$ by assuming the ansatz $\varphi_{2}(p) \propto\left(p^{2}+1\right)^{-3 / 2}+\eta\left(p^{2}+\frac{1}{4}\right)\left(p^{2}+1\right)^{-5 / 2}$ for the nonvanishing component of the Salpeter amplitude (5) to describe massless pseudoscalar bound states of fermions with mass $m=0$, $V(r)=-N(r) / D(r)$ with the two abbreviations $D(r) \equiv 2 r\left[4(1+\eta) K_{0}(r)-\eta r K_{1}(r)\right]$ and $N(r) \equiv \pi\left[4+\eta\left(4+r^{2}\right)\right]\left[I_{0}(r)-\mathbf{L}_{0}(r)\right]+\pi(4+5 \eta) r\left[I_{1}(r)-\mathbf{L}_{1}(r)\right]-4(2+3 \eta) r$ for numerator and denominator, respectively, for a few $\eta$-parameter choices from the interval $-1 \leq \eta \leq 0$ : $\eta=0$ (black solid line, again [6, Sec. 5.A]), $\eta=-0.25$ (red dotted line), $\eta=-0.5$ (magenta short-dashed line), $\eta=-0.75$ (blue long-dashed line), and $\eta=-1$ (violet dot-dashed line).

- For $\eta<-1$ (Fig. 6), the jump of $V(r)$ at its discontinuity flips for $\widetilde{\eta} \approx-1.463012572$ :

- For $\widetilde{\eta}<\eta<-1, V(r)$ remains strictly negative up to $r_{0}$, performs a $-\infty$ to $+\infty$ jump and approaches in the limit $r \rightarrow \infty$, via a local minimum, zero from below.

- For $-\infty<\eta<\widetilde{\eta}, V(r)$ rises with $r$ at its inevitable jump discontinuity at $r=r_{0}$ to $+\infty$, succumbs to a fall to $-\infty$ and resumes its rise to zero in the far distance.

We are led to conclude that a proper incorporation of confinement, manifesting itself by the presence of an infinite discontinuity at the location of the zero $r_{0}$ of the configuration-space Salpeter amplitude $\varphi(r)$, entails a drastic alteration of the physical impact of the extracted potential $V(r)$ : whereas in Ref. [6], starting from a Salpeter-amplitude ansatz equivalent to the special case of a vanishing admixture of the second term in our present ansatz (12), i.e., for $\eta=0$, we observed that not only for $m=\mu>0$ but, in fact, for all $m \geq \mu$ the extracted potential is not confining, here we obtain also for quarks with nonzero mass, at least for the case $m=\mu$, confinement for any mixing $\eta \notin[-1,0]$ enabling the existence of a zero of $\varphi(r)$. 


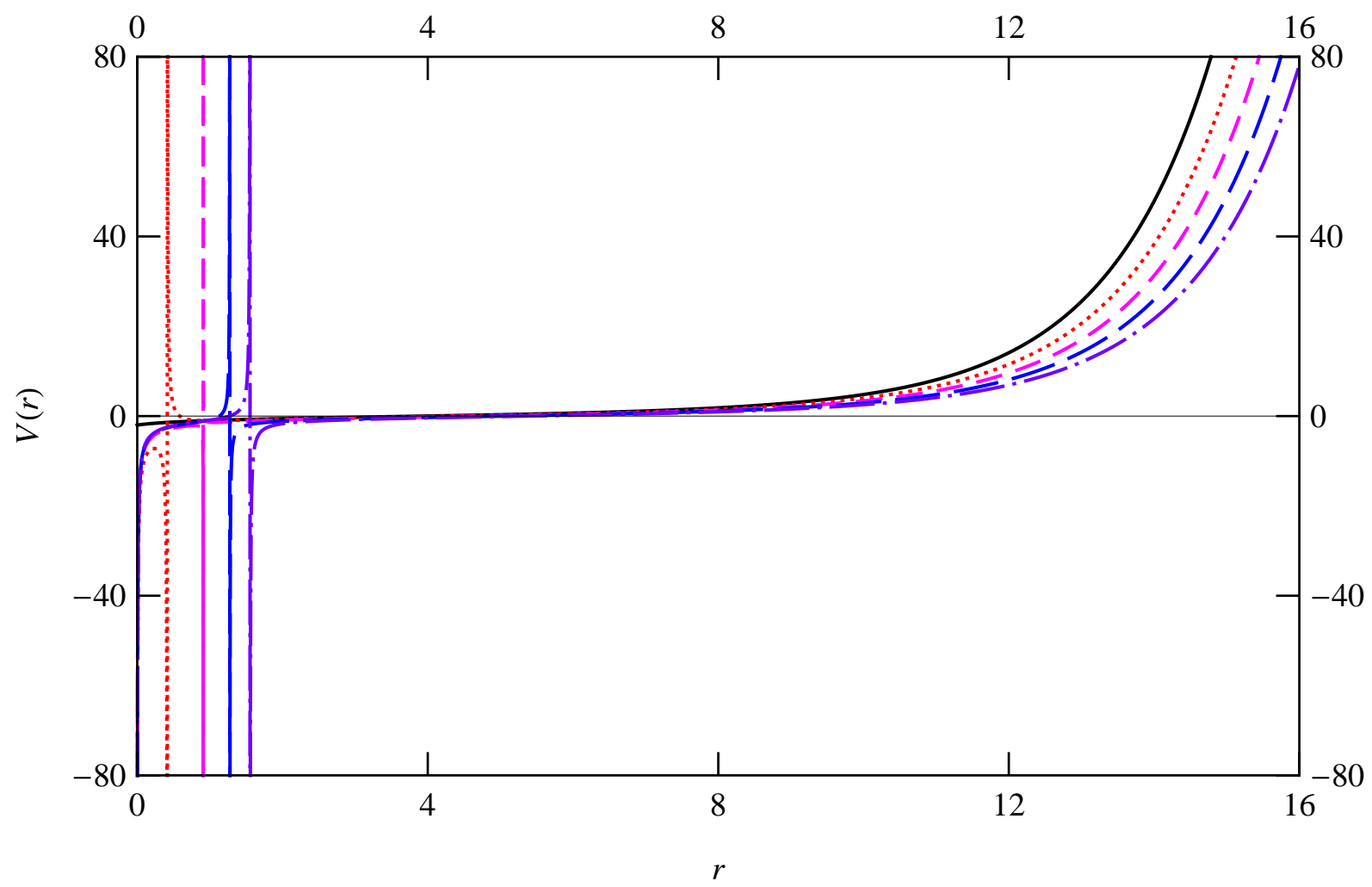

Figure 4: Configuration-space potential $V(r)$ extracted from the Salpeter equation (3) with interaction-kernel Lorentz structure $2 \Gamma \otimes \Gamma=\gamma_{\mu} \otimes \gamma^{\mu}+\gamma_{5} \otimes \gamma_{5}-1 \otimes 1$ by assuming the ansatz $\varphi_{2}(p) \propto\left(p^{2}+1\right)^{-3 / 2}+\eta\left(p^{2}+\frac{1}{4}\right)\left(p^{2}+1\right)^{-5 / 2}$ for the nonvanishing component of the Salpeter amplitude (5) to describe massless pseudoscalar bound states of fermions with mass $m=0$, $V(r)=-N(r) / D(r)$ with the two abbreviations $D(r) \equiv 2 r\left[4(1+\eta) K_{0}(r)-\eta r K_{1}(r)\right]$ and $N(r) \equiv \pi\left[4+\eta\left(4+r^{2}\right)\right]\left[I_{0}(r)-\mathbf{L}_{0}(r)\right]+\pi(4+5 \eta) r\left[I_{1}(r)-\mathbf{L}_{1}(r)\right]-4(2+3 \eta) r$ for numerator and denominator, respectively, for a few $\eta$-parameter values from the range $-\infty<\eta \leq-1$ : $\eta=-1$ (black solid line, as a benchmark), $\eta=-1.25$ (red dotted line), $\eta=-1.5$ (magenta short-dashed line), $\eta=-1.75$ (blue long-dashed line), and $\eta=-2$ (violet dot-dashed line).

\section{Summary and Discussion of Findings, and Outlook}

In the present study, we demonstrated, within an instantaneous Bethe-Salpeter formalism, that it is achievable to formulate, for pseudoscalar mesons, exact analytical solutions of the homogeneous Bethe-Salpeter equation for quark-antiquark bound states that exhibit both the absolute confinement demanded from coloured degrees of freedom and the masslessness expected for all Goldstone bosons related to spontaneously broken continuous symmetries, in the sense of establishing a rigorous relationship between tentatively postulated solutions and the form of the effective interaction responsible for the formation of such bound states.

By inversion, we harvest the effective interactions in the disguise of configuration-space potentials that carry an imprint of confinement: ${ }^{2}$ The absence of free coloured states entails

\footnotetext{
${ }^{2}$ One might wonder whether such a potential provides absolute confinement. However: Due to the rise of the potential to infinity in suitable intervals adjacent to the off-origin discontinuity, we may take as granted that any transmission coefficient for the corresponding Schrödinger problem vanishes. Since the relativistic
} 


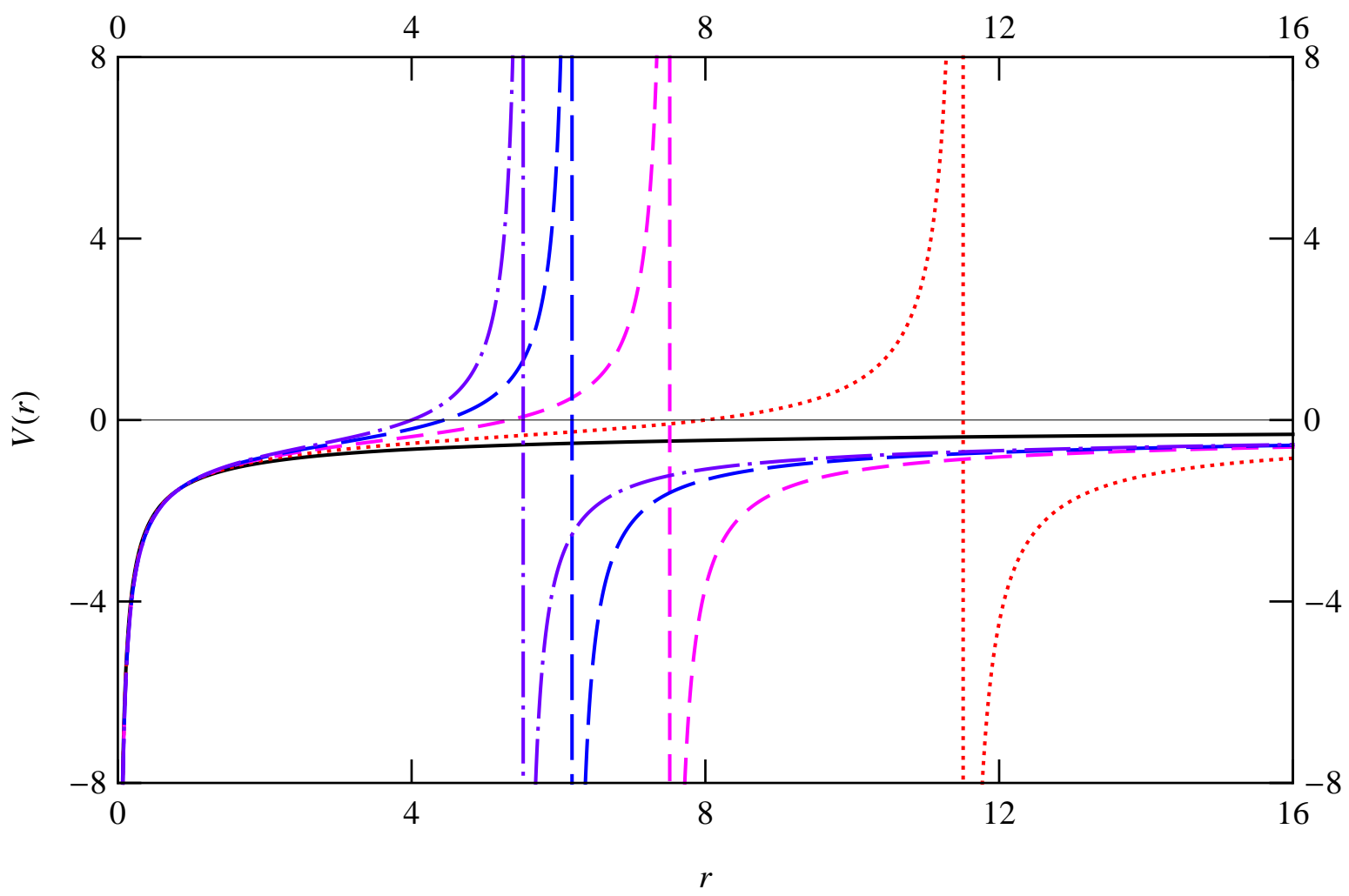

Figure 5: Configuration-space potential $V(r)$ extracted from the Salpeter equation (3) with interaction-kernel Lorentz structure $2 \Gamma \otimes \Gamma=\gamma_{\mu} \otimes \gamma^{\mu}+\gamma_{5} \otimes \gamma_{5}-1 \otimes 1$ by assuming the ansatz $\varphi_{2}(p) \propto\left(p^{2}+1\right)^{-3 / 2}+\eta\left(p^{2}+\frac{1}{4}\right)\left(p^{2}+1\right)^{-5 / 2}$ for the nonvanishing component of the Salpeter amplitude (5) to describe massless pseudoscalar bound states of fermions with mass $m=1$ : $V(r)=-\{\pi[8+\eta(8-3 r)] \exp (-r)\} /\left\{4 r\left[4(1+\eta) K_{0}(r)-\eta r K_{1}(r)\right]\right\}$, for the values $\eta=0$ (black solid line [6, Sec. 5.B]), $\eta=0.5$ (red dotted line), $\eta=1$ (magenta short-dashed line), $\eta=1.5$ (blue long-dashed line) and $\eta=2$ (violet dot-dashed line) of our mixing parameter.

a zero of the bound-state wave function, which causes the potentials to rise without limit at finite interquark distance and thus appears as the crucial ingredient of the present scenario.

Needless to say, our next step has to be to leave the comparatively safe realm of analytic investigations and to exploit the explicit findings for the quark mass functions derived from phenomenologically reliable QCD-based models within the framework of Dyson-Schwinger equations existing in the literature (unfortunately, however, available at present only as the results of numerical computations) in order to obtain a still more realistic understanding of the way the strong interactions enter in the Salpeter approach to pions and kaons [18]. Such analysis might even culminate in an attempt to approximately reconstruct the shape of the Bethe-Salpeter amplitudes for light pseudoscalar mesons in form of an analytic expression.

kinetic energy is bounded from above by its nonrelativistic counterpart, entering the Schrödinger equation, we expect that the transmission coefficient of a spinless Salpeter equation with such potential vanishes too. 


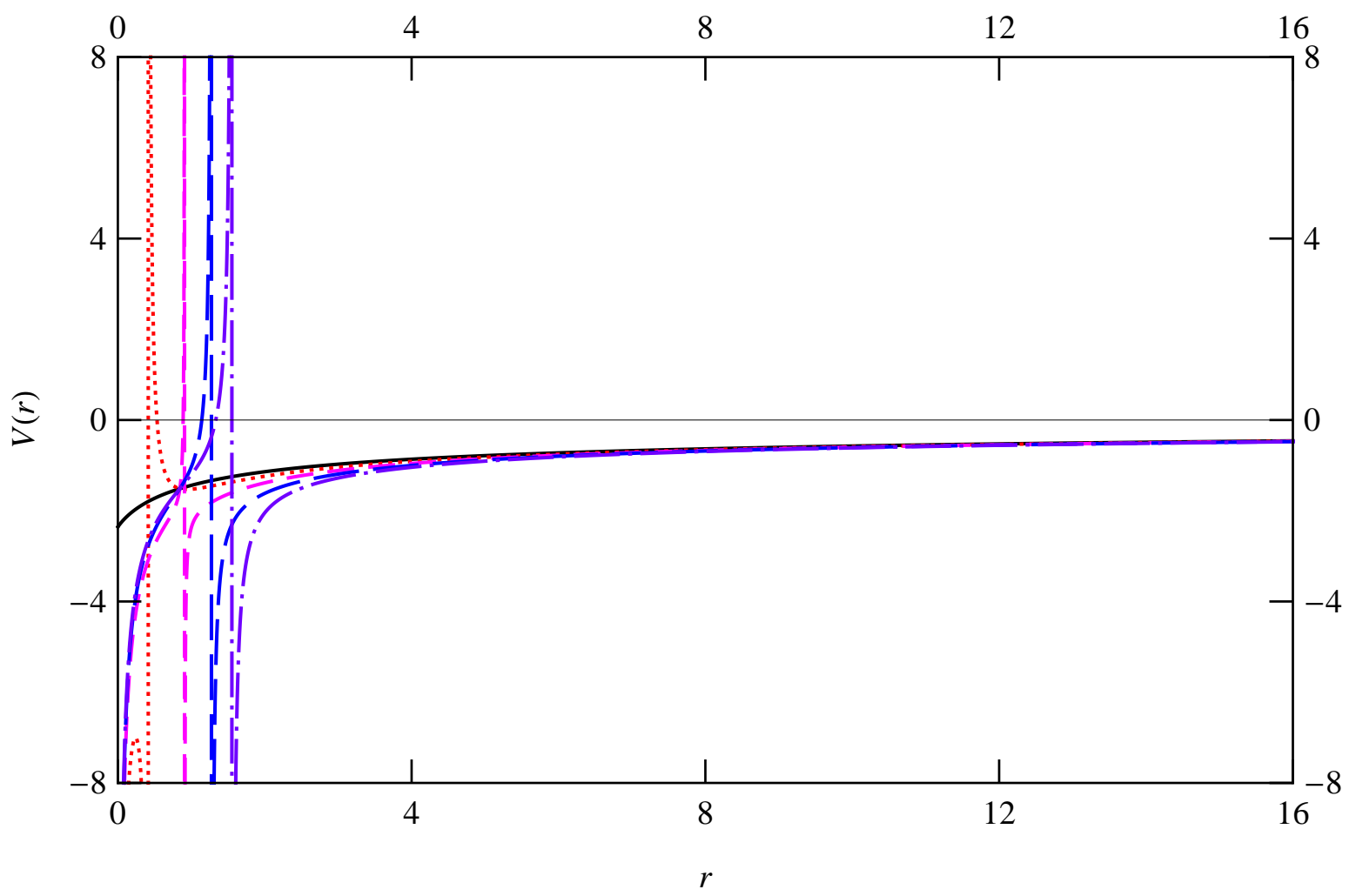

Figure 6: Configuration-space potential $V(r)$ extracted from the Salpeter equation (3) with interaction-kernel Lorentz structure $2 \Gamma \otimes \Gamma=\gamma_{\mu} \otimes \gamma^{\mu}+\gamma_{5} \otimes \gamma_{5}-1 \otimes 1$ by assuming the ansatz $\varphi_{2}(p) \propto\left(p^{2}+1\right)^{-3 / 2}+\eta\left(p^{2}+\frac{1}{4}\right)\left(p^{2}+1\right)^{-5 / 2}$ for the nonvanishing component of the Salpeter amplitude (5) to describe massless pseudoscalar bound states of fermions with mass $m=1$, $V(r)=-\{\pi[8+\eta(8-3 r)] \exp (-r)\} /\left\{4 r\left[4(1+\eta) K_{0}(r)-\eta r K_{1}(r)\right]\right\}$, for "confining," yet negative mixing: $\eta=-1$ (black solid line), $\eta=-1.25$ (red dotted line), $\eta=-1.5$ (magenta short-dashed line), $\eta=-1.75$ (blue long-dashed line), and $\eta=-2$ (violet dot-dashed line).

\section{Acknowledgements}

We are grateful to Lei Chang for drawing our attention to an implication of the violation of the axiom of reflection positivity for the behaviour of confining Bethe-Salpeter amplitudes.

\section{References}

[1] H. A. Bethe and E. E. Salpeter, Phys. Rev. 82 (1951) 309;

M. Gell-Mann and F. Low, Phys. Rev. 84 (1951) 350;

E. E. Salpeter and H. A. Bethe, Phys. Rev. 84 (1951) 1232.

[2] W. Lucha and F. F. Schöberl, J. Phys. G: Nucl. Part. Phys. 31 (2005) 1133, arXiv:hepth/0507281.

[3] E. E. Salpeter, Phys. Rev. 87 (1952) 328.

[4] W. Lucha and F. F. Schöberl, Phys. Rev. D 87 (2013) 016009, arXiv:1211.4716 [hep-ph]. 
[5] W. Lucha, Proc. Sci., EPS-HEP 2013 (2013) 007, arXiv:1308.3130 [hep-ph].

[6] W. Lucha and F. F. Schöberl, Phys. Rev. D 92 (2015) 076005, arXiv:1508.02951 [hep-ph].

[7] Z.-F. Li, W. Lucha, and F. F. Schöberl, Phys. Rev. D 76 (2007) 125028, arXiv:0707.3202 [hep-ph].

[8] J.-F. Lagaë, Phys. Rev. D 45 (1992) 305.

[9] M. G. Olsson, S. Veseli, and K. Williams, Phys. Rev. D 52 (1995) 5141, arXiv:hep$\mathrm{ph} / 9503477$.

[10] Handbook of Mathematical Functions, edited by M. Abramowitz and I. A. Stegun (Dover, New York, 1964).

[11] W. Lucha and F. F. Schöberl, Int. J. Mod. Phys. A 07 (1992) 6431; in Proceedings of the International Conference on Quark Confinement and the Hadron Spectrum, edited by N. Brambilla and G. M. Prosperi (World Scientific, River Edge, NJ, 1995) p. 100, arXiv:hepph/9410221; Int. J. Mod. Phys. A 14 (1999) 2309, arXiv:hep-ph/9812368; Fizika B 8 (1999) 193, arXiv:hep-ph/9812526; Recent Res. Devel. Phys. 5 (2004) 1423, arXiv:hep-ph/0408184.

[12] P. Maris, C. D. Roberts, and P. C. Tandy, Phys. Lett. B 420 (1998) 267, arXiv:nuclth/9707003.

[13] P. Maris and C. D. Roberts, Phys. Rev. C 56 (1997) 3369, arXiv:nucl-th/9708029.

[14] J. Glimm and A. Jaffe, Quantum Physics - A Functional Integral Point of View (Springer, New York, 1981).

[15] C. D. Roberts, A. G. Williams, and G. Krein, Int. J. Mod. Phys. A 07 (1992) 5607.

[16] C. D. Roberts, Prog. Part. Nucl. Phys. 61 (2008) 50, arXiv:0712.0633 [nucl-th].

[17] K. Osterwalder and R. Schrader, Commun. Math. Phys. 31 (1973) 83; 42 (1975) 281.

[18] W. Lucha and F. F. Schöberl, in preparation. 